\title{
Cellular pseudosarcomatous fibroepithelial stromal polyp of the cervix: a lesion mimicking as sarcoma
}

\begin{abstract}
Introduction: Fibroepithelial stromal polyps (FESP) are infrequent mesenchymal lesions found in vulvovaginal region but may also occur in the cervix, usually seen in reproductive age group. FESP exhibiting bizarre cytomorphology, atypical mitoses, or hypercellularity, raises the possibility of malignancy and continue to be underrecognized.
\end{abstract}

Case summary: We report a case of 45 years old, postmenopausal female presented with a large polypoidal cauliflower like growth with ulcerated surface, arising from exocervix. Cinical diagnosis of cervical malignancy was suggested. Histopathological examination revealed a polypoidal tumor mass composed of cellular fibrovascular stroma covered with stratified squamous epithelium. There are areas showing marked hypercellularity, bizarre cytomorphology and frequent mitotic figures $(>10 / 10 \mathrm{HPF})$ including atypical ones. Important morphologic clues to the diagnosis of FESP were its superficial location, lack of an identifiable lesional margin, extension of abnormal stromal tissue up to the mucosal-submucosal interface (absence of cambium layer), presence of characteristic stellate cells and the individually scattered multinucleate stromal cells. Before making the diagnosis of Cellular pseudosarcomatous FESP we excluded other differentials like Anngiomyxoma, Angiomyofibroblastom, Botyroid embryonal rhabdomyosarcoma, Leiomyosarcoma, Low grade endometrial stromal sarcoma and malignant peripheral nerve sheath tumor.

Conclusion: Awareness of the spectrum of histologic features that these lesions can exhibit is essential to avoid unnecessary radical surgery or chemoradiation treatment.

Keywords: cellular, pseudosarcomatous, fibroepithelial, stromal polyps, cervix
Volume 3 Issue I - 2018

\author{
Mahboob Hasan' ,Ruquiya Afrose ${ }^{2}$,Mariya \\ Kamal' \\ 'Department of pathology, Aligarh Muslim University, India \\ ${ }^{2}$ Department of pathology, Uttar Pradesh University of Medical \\ Sciences, India
}

\begin{abstract}
Correspondence: Ruquiya Afrose, Assistant professor, Department of Pathology, Uttar Pradesh University of Medical Sciences, Saifai Etawah, 20630I, India, Tel 9219716166, Email ruqs.afrose@gmail.com
\end{abstract}

Received: November 17, 2016 | Published: February 21, 2018
Abbreviations: FESP, fibroepithelial stromal polyp; AMF, angiomyofibroblastoma; ERMS, embryonal rhabdomyosarcoma; ESS, endometrial stromal sarcoma; MPNST, malignant peripheral nerve sheath tumor

\section{Introduction}

The fibroepithelial stromal polyp (FESP) are infrequent mesenchymal lesions of distal female genital tract found in vulvovaginal region but infrequently may also occur in the cervix. ${ }^{1-3}$ These arise from subepithelial myxoid stroma and represent a reactive process rather than neoplastic one. ${ }^{3}$ Many cases are seen associated with pregnancy, suggesting its association with hormones. Because of bizarre cytomorphology, atypical mitoses, and hypercellularity, FESP raises the possibility of malignancy and continue to be underrecognized. Here we are presenting an unusual case of pseudosarcomatous FESP of cervix in a postmenopausal female exhibiting metaplastic bone formation.

\section{Case report}

A 45 years old, postmenopausal female presented with a history of spotting and a large mass protruding from vagina for the last 1year. Per vaginal examination revealed a large polypoidal cauliflower like growth, arising from the cervical lip. Cinical suspicion of cervical malignancy was made and hence polyp was resected out and sent for histopathologic diagnosis. Gross examination revealed a cerebriform polypoidal growth measuring $6.5 \times 5 \times 3 \mathrm{~cm}$ with surface ulceration. At its attachment site part of cervical lip is also identified (Arrow) (Figure
1A). Cross section shows a solid homogenous grey white tumor with myxoid and gelatinous areas. Polyp is soft to firm in consistency with focal bony hard areas. Histopathological examination revealed a polypoidal tumor mass composed of cellular fibroblastic stroma and central fibrovascular core covered with variably thickened stratified squamous epithelium (Figure 1B). Stroma varies from edematous and hypocellular with bland spindle-shaped cells (Figure 1C), indistinct cytoplasm to areas that are hypercellular and contain cells with markedly enlarged hyperchromatic nuclei, bizarre cytomorphology and frequent mitotic figures $(>10 / 10 \mathrm{HPF})$ including atypical ones (Figure 1D) (Figure 1E). Tumor cells are extending up to the overlying squamous epithelium and on periphery metaplastic bone formation is seen (Figure 1F). Although surface of the polyp is ulcerated showing inflammatory exudates and fibrinoid necrosis, no area of necrosis or haemorrhage is seen within the tumor. On immunohistochemistry these stromal cells are showing diffuse and strong positivity for desmin and estrogen receptor (Figure 1F) (Figure 1G). While negative for smooth muscle actin, CD34 and S100. Important morphologic clues to the diagnosis of FESP were its superficial location, lack of an identifiable lesional margin, extension of abnormal stromal tissue up to the mucosal-submucosal interface (absence of cambium layer), presence of characteristic stellate cells and the occasionally scattered individually multinucleate stromal cells. Before making the diagnosis of cellular pseudosarcomatous FESP we excluded other differentials like Anngiomyxoma, Angiomyofibroblastom, Botyroid embryonal rhabdomyosarcoma, Leiomyosarcoma, Low grade endometrial stromal sarcoma and malignant peripheral nerve sheath tumor. 


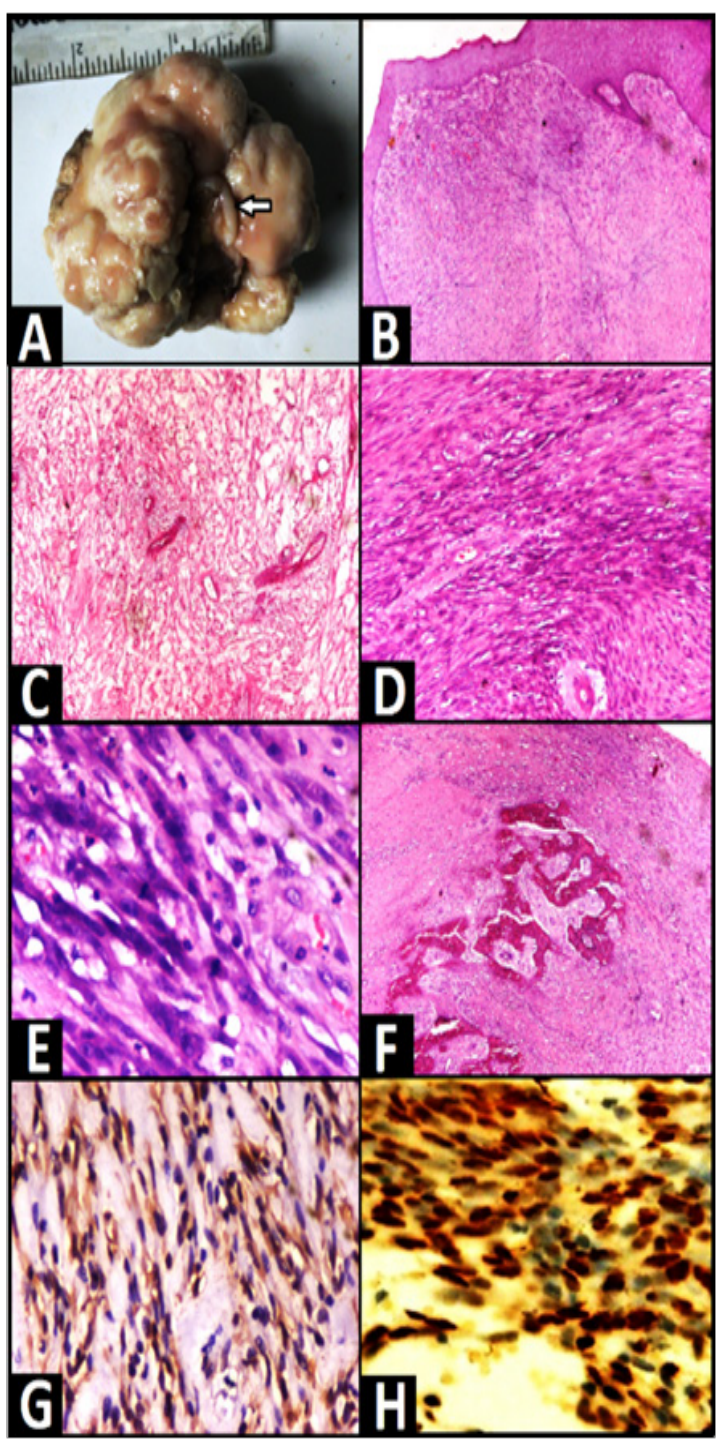

Figure I (A) Cerebriform polypoidal growth with surface ulceration. part of cervical lip is also identified (Arrow).

(B) Cellular fibroblastic stroma with variably thickened stratified squamous epithelium.

(C) Oedematous and hypocellular myxoid stroma with bland spindle cell morphology.

(D \& E) Hypercellular mitotically active stroma with markedly enlarged hyperchromatic and pleomorphic nuclei.

(F) Metaplastic bone formation at periphery.

( $G$ \& H) Diffuse and strong immuno positivity for desmin and estrogen receptor.

\section{Discussion}

FESP are uncommon benign stromal polyp of reproductive age group involving distal female genital tract, most commonly vagina followed by vulva and rarely cervix. ${ }^{1-3}$ Involvement of other site like renal pelvis ${ }^{4}$ and cutaneous lesions ${ }^{5}$ have also been reported. Involvement of cervix, especially in postmenopausal women is very rare and said to be associated with hormone therapy. ${ }^{2,3,6}$
The pathogenesis of FESP is not well understood, however these benign lesions seem to represent a reactive hyperplastic process which involves the distinctive subepithelial stroma of the lower female genital tract rather than a true neoplasm. This is based on the facts that these lesions have no clearly defined margin. ${ }^{3}$ Another hypothesis is based on the finding that the stromal cells of FESP can express estrogen and progesterone receptors, suggesting the role of these hormones in the pathogenesis of this lesion. ${ }^{3}$ In our case also estrogen receptor positivity is seen. Nucci et al. ${ }^{3}$ studied 33 cases of fibroepithelial stromal polyps which showed marked hypercellularity, cytologic pleomorphism, mitotic counts of more than 10 mitoses per 10 high-power fields, and the presence of atypical mitoses. On immunohistochemically, seven out of 12 cases were desmin positive and one out of 11 cases was smooth muscle actin positive. Involved sites were the vagina (18 of 33), cervix ( 7 of 33), and vulva ( 8 of 33). ${ }^{3}$ Before making the diagnosis of Cellular pseudosarcomatous FESP we excluded other differentials like Aggressive Angiomyxoma, Angiomyofibroblastom, Botyroid embryonal rhabdomyosarcoma, Leiomyosarcoma, Low grade endometrial stromal sarcoma and malignant peripheral nerve sheath tumor. Aggressive angiomyxoma (AA) may be considered as a possibility due to presence of edematous stroma in FESP. AA occurs in the vulvovaginal and perineal area in women and is a locally aggressive, nonmetastasizing lesion with a tendency to recur if incompletely excised. ${ }^{6,7} \mathrm{AA}$ is less polypoidal, more deep in location and exhibits a prominent vascular pattern distributed more diffusely throughout the tumor. Another differentiating feature not seen in FESP is that AA tends to have myoid bundles cuffing the vascular component. ${ }^{6}$ Cellular FESP often exhibit mitotic activity and contain atypical stromal cells not usually seen in AA. Both are often desmin positive so cannot be differentiated on immunoperoxidase staining. Another differential diagnosis of FESP is Angiomyofibroblastoma (AMF) which is a benign tumor of the vulvovaginal region. ${ }^{8,9}$ Sarcomatous transformation of AMF has rarely been reported. ${ }^{10}$ Cellular FESP is a polypoid mass whereas AMF is a well-circumscribed submucosal nodule. The stromal cells of AMF characteristically cluster around the vasculature which is not prominently seen in cellular FESP. ${ }^{3}$ Multinucleate cells and plump epithelioid cells can be seen in both cellular FESP and AMF; nuclear atypia is however absent in AMF. Large thick-wall and often hyalinized vessels are seen in cellular FESP as opposed to the vasculature of AMF which tends to be composed of vessels which have more delicate walls. Both are often desmin positive so there is no role of immunohistochemistry.

FESP occasionally exhibit a grape like appearance resembling Botryoid embryonal rhabdomyosarcoma (ERMS). ${ }^{11}$ It involves mucosal surfaces containing neoplastic cells extending up to the epithelial stromal interface. ERMS is a tumor of early childhood, whereas cellular FESP usually occur in women of reproductive age group. Histologically, botryoid ERMS exhibits a submucosal hypercellular zone (cambium layer) whereas FESP usually exhibit increased cellularity toward the center of the lesion with decreased cellularity near the epithelial stromal interface. The lack of rhabdomyoblasts, myoglobin, fast myosin, and myogenin can help distinguish cellular FESP from ERMS. Cellular FESP sometimes exhibits a fascicular architecture, along with an increased mitotic rate, atypical cells, and atypical mitoses which can raise the possibility of LMS. Identifiable lesional margin and a polypoidal appearance are features not found in LMS. The tumor cells of LMS tend to exhibit features of smooth muscle differentiation with elongated nuclei in a 
characteristic "box-car" or "cigar" shape and abundant eosinophilic cytoplasm. The stromal cells of cellular FSP are usually desmin positive and sometimes actin positive. ${ }^{3,12}$ Low-grade endometrial stromal sarcoma (ESS) usually involve endometrial cavity but can secondarily involve the cervix and can metastasize to the vulva and vagina. Similar to FESP, ESS can be polypoid and highly vascular. The vessels, however, seen in ESS resemble spiral arterioles while that of cellular FSP are often variable in size and can be thick walled and hyalinized. Central vascular core is often present in cellular FSP, whereas absent in ESS. Thick hyalinized collagen bands in a starburst pattern, are characteristically seen in some ESS, are absent in cellular FSP. However stromal atypia is not a feature of ESS, which is commonly present in FESP. On immunochemistry some ESS are desmin and/or keratin positive in a do like perinuclear pattern, whereas negative in cellular FESP. ${ }^{13}$ Some cellular FESP show wavy and tapered spindle cells, giving it a neural-like appearance, resembling malignant peripheral nerve sheath tumor (MPNST) specially when tumor is mitotically active. MPNST can occur rarely in the cervix ${ }^{14}$ and vulva. ${ }^{15}$ Both these lesions can be polypoid; but cellular FESP is more vascular, with thick walled and larger caliber vessels. In MPNST, perivascular accentuation is commonly seen which is absent in cellular FESP. On immunohistochemistry focal staining for S-100 is seen in approximately $50 \%$ of MPNST. In summary, cellular FESP of the lower female genital tract can exhibit a spectrum of bizarre cytomorphologic features, atypical mitosis and hypercellularity, FESP raises possibility of malignancy and continues to be underrecognised. Awareness of the spectrum of histologic features that these lesions can exhibit is essential to avoid unnecessary overtreatment.

\section{Acknowledgements}

None.

\section{Conflict of Interest}

The author declares no conflict of interest.

\section{References}

1. Nielsen GP, Young RH. Mesenchymal tumors and tumor-like lesions of the female genital tract: a selective review with emphasis on recently described entities. Int J Gynecol Pathol. 2001;20(2):105-127.

2. Santos LD, Ng A, Tan YM. Cellular pseudosarcomatous fibroepithelial stromal polyp of the endocervix. Pathology. 2004;36(4):376-378.
3. Nucci MR, Young RH, Fletcher CD. Cellular pseudosarcomatous fibroepithelial stromal polyps of the lower female genital tract: an underrecognized lesion often misdiagnosed as sarcoma. Am J Surg Pathol. 2000;24(2):231-240.

4. Parada D, Moreira O, Gledhill T, et al. Cellular pseudosarcomatous fibroepithelial stromal polyp of the renal pelvis. APMIS. 2005;113(1):7074.

5. Cathro HP, Patterson JW, Wick MR. Cutaneous pseudosarcomatous polyp: a recently described lesion. Ann Diagn Pathol. 2008;12(6):440444

6. Granter SG, Nucci MR, Fletcher CDM. Aggressive angiomyxoma: reappraisal of its relationship to angiomyofibroblastoma in a series of 13 cases. Histopathology. 1997;30(1):3-10.

7. Steeper TA, Rosai J. Aggressive angiomyxoma of the female pelvis and perineum: report of nine cases of a distinctive type of gynecologic soft tissue neoplasm. Am J Surg Pathol. 1983;7(5):463-475.

8. Fletcher CDM, Tsang WYW, Fisher C, et al. Angiomyofibroblastoma of the vulva. A benign neoplasm distinct from aggressive angiomyxoma. Am J Surg Pathol. 1992;16(4):373-382.

9. Laskin WB, Fetsch JF, Tavassoli FA. Angiomyofibroblastoma of the female genital tract: analysis of 17 cases including a lipomatous variant. Hum Pathol. 1997;28(9):1046-1055.

10. Nielsen GP, Young RH, Dickersin GR, et al. Angiomyofibroblastoma of the vulva with sarcomatous transformation ("angiomyofibrosarcoma"). Am J Surg Pathol. 1997;21(9):1104-1108.

11. Elliott GB, Reynolds HA, Fidler HK. Pseudo-sarcoma botryoides of cervix and vagina in pregnancy. J Obstet Gynaecol Br Commonw. 1967;74(5):728-733.

12. Hartmann CA, Sperling M, Stein H. So-called fibroepithelial polyps of the vagina exhibiting an unusual but uniform antigen profile characterized by expression of desmin and steroid hormone receptors but no muscle-specific actin or macrophage markers. Am J Clin Pathol. 1990;93(5):604-608.

13. Song JS, Song DE, Kim K-R, et al. Cellular Pseudosarcomatous Fibroepithelial Stromal Polyp of the Vagina during Pregnancy: A lesion that is overdiagnosed as a malignant tumor. Korean journal of pathology. 2012;46(5):494-498.

14. Keel SB, Clement PB, Prat J, et al. Malignant schwannoma of the uterine cervix: a study of three cases. Int J Gynecol Pathol. 1998;17(3):223230 .

15. Lawrence WD, Shingleton HM. Malignant schwannoma of the vulva: a light and electron microscopic study. Gynecol Oncol. 1978;6(6):527537. 